\title{
Society for Pediatric Research 2010 Presidential Address: Academic Pediatrics and the Narrative of Discovery
}

\author{
DAVID N. CORNFIELD \\ Department of Pediatrics, Stanford University School of Medicine, Stanford, California 94305
}

\section{PURVIEW OF ACADEMIC PEDIATRICS}

Supreme Court Justice Louis Brandeis called medicine a "learned profession; a profession responsible for a body of knowledge, a meaningful amount of which must be gained from experience (1)." In this construct, experience must have currency. Moreover, the profession is responsible for both advancing and communicating the knowledge to the next generation. Intrinsic to pediatrics is a code of ethics that entails service to others, articulating and enforcing standards, and promoting a culture that values service above reward. This social contract, between society and medicine, confers both respect and autonomy to medicine. In no small part, our collective mindfulness of this contract has enabled pediatrics to enjoy a relatively generous amount of both respect and autonomy, even in the present challenging times.

\section{JOY, PRIVILEGE OF MEDICINE}

As pediatricians, we have chosen to work in one way or another, on behalf of child health and welfare. Our work grants us the privilege and joy of being gifted with a precious and sometimes fragile trust. Each of us, in our own way, is ennobled by striving to be a bit better tomorrow than we are today. Over many years, as our reach exceeds our grasp, the humanity of those engaged in academic pediatrics is heightened. Although the merits and critical determinants of, as well as the impediments to, the success of an academic pediatrician are straight forward, the question remains as to how might these goals best be accomplished?

\section{GENERATIONAL IMPERATIVE}

As we begin to consider strategies to overcome the considerable challenges to academic pediatrics, we ought be comforted by the notion that each generation possesses a sense of urgency, struggles with seemingly entirely new problems that threaten the entire field, and yet finds a sustainable solution.

Received June 2, 2011; accepted June 29, 2011.

Correspondence: David N. Cornfield, M.D., Department of Pediatrics, Stanford University School of Medicine, 701 Welch Road, Room 3328, Bldg A, Stanford, CA 94305; e-mail: dcornfield@stanford.edu

Presented at the 2010 Annual Meeting of the Pediatric Academic Societies, Vancouver, British Columbia, Canada.
To contextualize the present challenges, it might be useful to recognize that pediatrics has gone through relatively distinct epochs of progress. At the outset, in the mid-19th century, pediatrics was devoted to creating a justification for children's doctors. With the establishment of clear evidence of need and an appreciation by the public that pediatrics merited inclusion as a distinct field, the presence of physicians dedicated to children motivated the rapid acceleration in the body of knowledge specific to children. With advent of the antibiotic era, heralded by the introduction of penicillin and sulfa drugs in the 1940s, the knowledge proved critical as pediatrics was suddenly invested with the power to cure infections in children using antibiotics, public health principles, and vaccines (Fig. 1) (2).

In 1970, pediatrics entered the era of child development. The discipline began to place greater emphasis upon the nuances of child development, physiologic, behavioral, and pathophysiologic (3). And presently, we find ourselves in the era of acute on chronic illness. Survivors of prematurity, serious acute illness and chronic illnesses that introduce great morbidity and intensity into everyday life, represent a new care paradigm, with a large component of resources focused upon a relatively small number of children. This era results from the remarkable advances in the care that we, as a discipline, can bring to bear to support even the most critically ill child. We find ourselves in the era of acute on chronic disease survivors of serious illness facing life-long challenges, the cost of cure, and the increase of the medical home (4).

How is it that pediatrics, a specialty, an art, a calling, and discipline, has been able to craft an identity that transcends spatial and temporal constraints? In the next few minutes, I hope to articulate thematic principles that will answer the question and perhaps provide guidance for the future.

\section{ACADEMIC PEDIATRICS-CHALLENGES ON ALL FRONTS}

At present, academic pediatrics faces grave challenges. As illustrated in Figure 2, the core components of our mission are increasingly mutually challenged, each competing with the other for time, money, energy, and cognition. The core components of our mission patient care, research, education, and

Abbreviations: ARDS, acute RDS; CF, cystic fibrosis 


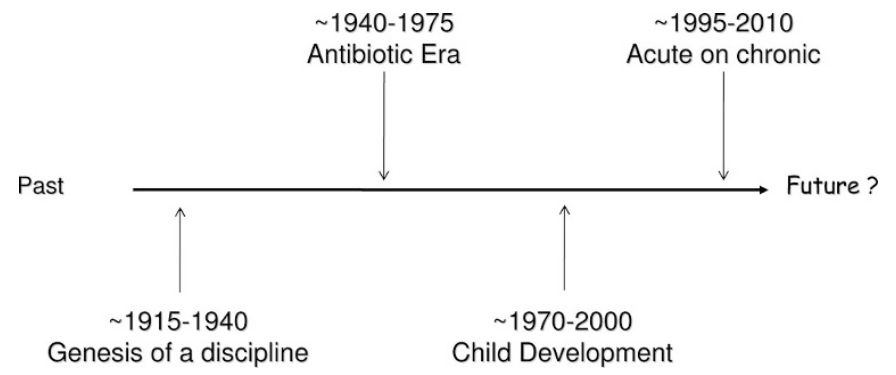

Figure 1. Epochs of pediatric medicine. Despite distinct epochal challenges, pediatrics has a singular identity. How?

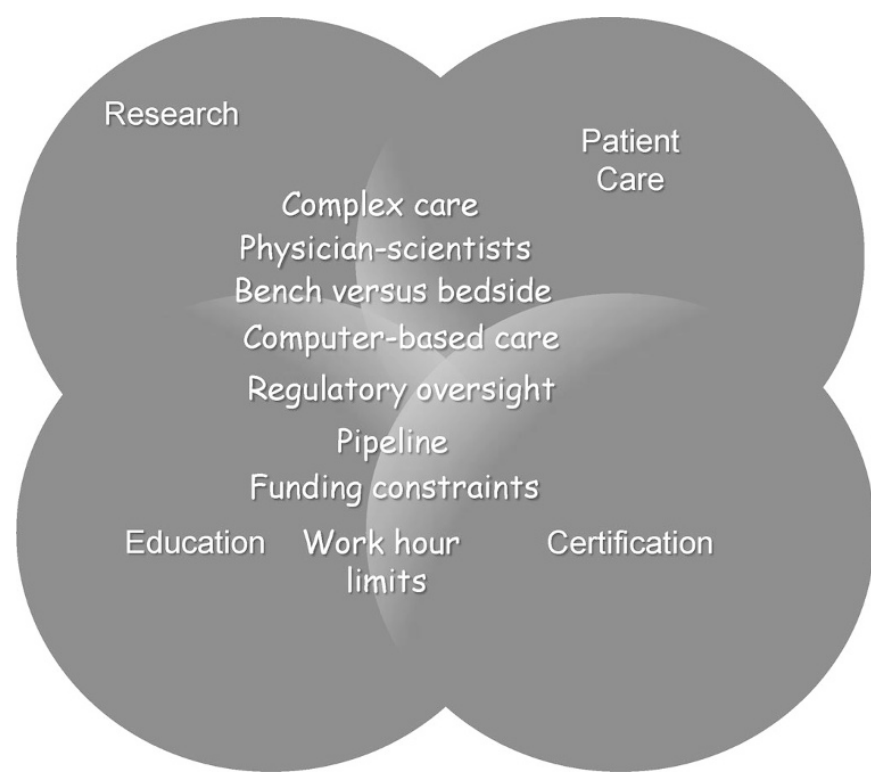

Figure 2. Challenges to academic pediatrics. The core missions of academic pediatrics, research, training, care delivery, and certification are increasingly challenged. Each challenge has an impact of multiple aspects of the core mission.

certification have become much more time-intensive. From the educational perspective, the challenges are fierce and include work-hour limitations, dramatically heightened levels of oversight for trainees, and a need to demonstrate educational competencies that may or may not favorably influence either training or patient care (4). Perhaps, most challenging and inarguably onerous is the remarkable administrative burden imposed on virtually all training programs, irrespective of size, by the American College of Graduate Medical Education (ACGME) (5). From the perspective of the discovery process, challenges include the ever-increasing time until scientific independence; the paradoxical separation between bench and bedside, despite the availability of powerful tools that can effectively and efficiently bridge the gap between patient care and discovery; and finally, the increasingly narrow perspective that high-intensity, highly specialized medicine demands $(6,7)$.

At the core, pediatrics has always been about children. In hewing closely to this focus, pediatrics consistently serves most nobly and well. Several factors in the present care delivery models challenge the focus. First, care is more technical than at any time in our collective past, conferring distance between provider and patient. Moreover, maintaining longitudinal relationships with patients and families, interac- tions that inform and motivate every pediatrician, are challenged by the time-intensity, highly specialized care and the acute on chronic nature of pediatric illness. It is, therefore, increasingly difficult to move sincerely and deftly between the bench and bedside. Creating and maintaining human relationships in the clinical setting are further challenged by the advent of the electronic medical record. Patients are evaluated, orders written, care delivered, often without the benefit of more than a single-bedside interaction as the digital world does not require physical presence. How can we know our best course when we are increasingly removed from the very experiences that so excited us to pursue a career that is as much vocation as avocation?

Superimposed upon the challenges intrinsic to teaching, patient care and research, is the burden of oversight in every arena. Of course, oversight is essential, but it is the very thrust of the oversight that poses the greatest threat. In the educational arena, physicians no longer are determining the relative value of educational curriculum. Oftentimes, educators, with no specific expertise in medicine, are making decisions surrounding the optimal training experiences. In the arena of accreditation, the issues are similarly challenging. Construction of the Program Information Form as outlined by the ACGME requires many person hours. The resulting document often exceeds 100 pages. This regulatory burden is present even for subspecialty programs with as few as a single fellow. Similarly, in the arena of care delivery, oversight, work-hour limitations, competencies, the electronic medical record, all combine, arguably, to make patient care more time-intensive and threaten, in some manner, to place ever-greater distance between physician and patient, thereby moving pediatric science further from its primary motivating force, children.

To meet the present challenges, academic pediatrics will need to identify some guiding overarching principles. Experiences with pediatric illnesses, clinical medicine, might serve as a paradigm for developing strategies.

\section{CLINICAL MEDICINE AS AN ENGINE FOR DISCOVERY}

Personally, I have spent many hours at the bedside of critically ill children with acute RDS (ARDS). Notwithstanding the challenges of a patient with ARDS, rates of death are decreasing over time. Children with ARDS have also demonstrated a significant decrease in mortality over the past two decades, with reported rates of mortality in excess of $50 \%$ in the early 1990s and a subsequent decline to less than $30 \%$ in 2005 (8).

In cystic fibrosis (CF), as well as ARDS, survival rates are progressively increasing. In children with CF born between 1970 and 1973, median survival for children was 23 years of age. In dramatic contrast, the current predicted actuarial for all people living with CF in North America is 38 years of age. The change in prognosis, in a relatively short while, is dramatically positive (9).

On the basis of the marked improvement in prognosis in these two disease states, I propose the first principle that might to guide our path. In specific, clinical medicine can, and fact, 
should, drive discovery, create knowledge by promoting a bidirectional flow of information. These disease paradigms demonstrate clearly that progress can be realized through iterative testing of multiple hypotheses. Another insight derived from these disease models is that interaction between patient and investigator catalyzes hypothesis generation. Clinical experience provides important preliminary evidence that ultimately motivates knowledge creation. Hypothesis testing in multiple clinical venues, from varied vantage points, enables the creation of durable and robust knowledge.

\section{DISCOVERY IS INCREMENTAL}

By examining the narrative of the discoveries in each of these disease states, but especially in $\mathrm{CF}$, wherein substantial and progressive improvement occurred over several decades, another principle can be readily derived. In specific, knowledge discovery is most commonly incremental, not monumental. In both ARDS and CF, multiple factors account for the improvement in outcomes. Improved anti-infectives, greater attention to nutrition, a more standard approach to care, improved technology, and better drug delivery systems, all have contributed to the improvement in outcomes (10).

The narrative of discovery relative to ARDS provides yet another insight into the evolution of disease definition. As long ago as 1821, Laennec described idiopathic lung anasarcapulmonary edema without heart failure-in his classic text "A Treatise on Diseases of the Chest (11)." In 1925, Sir William Osler provided still further texture to the definition in his classic Medicine textbook by carefully considering etiology and pathophysiology in but a single, elegant sentence "uncontrolled septicemia leads to frothy pulmonary edema that resembles serum, not the sanguinous transudative edema fluid seen in dropsy or congestive heart failure" (12).

Interestingly, army surgeons learned a very great deal about both etiology and effective treatment strategies in caring for wounded soldiers during World War II in both North Africa and Italy. "Casualties with severe brain, thoracic, abdominal, and extremity trauma ... had persistent 'wet respiration,' were most difficult to resuscitate, withstood operation poorly, and had the highest mortality .... etiology ... ineffectual cough ... persistent bronchopulmonary transudates. Treatment included assisted cough, transbronchial aspiration, oxygenation, bronchoscopy and tracheostomy. To treat the most advanced form, an effectual hand-operated positive pressure oxygen machine was devised (13)." For reasons that remain unclear, these wonderful descriptions and strategies did not find significant traction in the medical literature. Of course, the publication cited above appeared in 1981, more than 35 years following the intrepid acts of these remarkable and insightful surgeons. Delay in publication is a profound reminder of the notion that without the written word, the narrative remains undiscoverable. In 1967, Ashbaugh et al. provided the seminal, comprehensive, and definitive description of ARDS. The definition included careful and explicit criteria, an alveolar to arteriolar gradient of less than 200, diffuse, bilateral disease, an identifiable insult (e.g. sepsis, trauma, and aspiration) within 7 days of developing a compromise in oxygenation and perhaps, most presciently, surfactant dysfunction (14).

\section{CONTEXT ENABLES DISCERNING JUDGMENTS}

To further underscore the importance of narrative, I turn to the narrative of real patient with ARDS. The medical description for the patient might have read as follows: 18-year-old male, s/p coarctation of aorta repair as an infant, and kidney transplantation (2001) presented with a complaint of shortness of breath, dyspnea, and low-grade fever. Date of admission was January 10, 2008, with discharge date of August 25, 2008. The admission diagnosis included adenoviral sepsis and respiratory failure; secondary diagnoses that the patient acquired during the course of the hospitalization included vocal cord paralysis, hypertension, pancreatitis, deep venous thrombosis, skin ulceration, and disseminated intravascular coagulation.

Contrast the medical narrative with the personal narrative provided by the patient. By hearing the patient's voice, caregivers might be more able to understand the perspective and intent of the patient and the family (15). "My name is Ori, and I am 18 years of age. Throughout life I have gone through multiple surgeries, a kidney transplant, and recently a viral infection that shut down almost every organ in my body. After the viral infection had gone down I focused my time on studying, in hopes of proving that anything is possible. Today, I have finished my high school and will soon start college. I enjoy life, doing exercises, and playing my clarinet. Being able to do these things continues to make life interesting for me. Most doctors did not believe these things were possible."

Among the lessons in the present case is that the narrative of illness provides context. Clearly, the definition of ARDS has evolved over time. The evolution underscores the importance of the narrative, from latin verb "narrare," to recount. The narrative in Medicine describes a sequence of events and entails perspective (16). Especially so in this era of the electronic medical record (17), it is important to remember that no narrative, strictly speaking, is interchangeable, every person tells a singularly unique story-both patients and providers. For any discipline, the narrative is undiscoverable in the absence of either the written or spoken word. Narrative of disease, patient, provider, and illness is essential. ARDS and CF both provide emphatic testimony to the importance of the narrative. Clearly, in any discipline, the narrative is undiscoverable without the written or spoken word.

As the narrative provides context, it enables discerning judgments. In the present case, were care providers to have rendered decisions without understanding course of the disease, the underlying cause, the trajectory of illness, and the family ethic, the outcome would certainly have been different. However, the clinicians were ever mindful of the context and that made a substantive difference for this young man and his family.

Although the general appreciation of the importance of context is growing, context is under threat, as never before. Communication occurs in shorter time intervals, with everdecreasing words. Our current world is populated by hypertext. Data are provided without context, an abstract is 
considered sufficient to confer expert status to the reader (18). For a learner to carefully read not only an abstract but perhaps the methods, discussion, and results is increasingly rare. Information becomes knowledge only when placed into context.

The advent and unremitting popularity of twitter is till further evidence of the importance of context is threatened. Consistent with this notion, is the ubiquitously present smart phone, a device that may actually enhance distractions on work rounds (19). In the clinical arena, context is still further challenged by computerized-provider order entry. Providers can enter orders remotely, even without necessarily conferring with people at the bedside of the patient. In the clinical arena, the advent of work-hour limitations will further constrain development of the longitudinal relationships are required for making context-based decisions (20). Thus, narrative weds the past to the future. With an effective, compelling, and lucid narrative, both context and a focus on humanity might be achieved.

\section{TRUE PROGRESS OCCURS AT THE MARGINS OF SEEMINGLY DISTINCT DISCIPLINES}

For many centuries, philosophers have struggled with the notion of definition and categorization. Both Aristotle and Bertrand Russell struggled with the "law of the excluded middle" wherein everything must either be or not be. Clearly, the present era demonstrates that tremendous overlap exists. In fact, the greatest progress occurs when disciplines previously thought to be distinct are brought into close approximation (21). The National Institutes of Health Roadmap places great emphasis on a multidisciplinary approach to research. Overall, this begs the question of whether the concept of distinct disciplines is obsolete. In medicine and science, traditional boundaries no longer seem rational. Neurology, Psychiatry, and Functional Radiology are all distinct areas but are all brought to bear in the arena of cognitive neurosciences. In the case of single-cell molecular diagnostics, work groups that include physicists, computational biologists, structural engineers, and molecular biologists have combined to bring rapid advances to the field (22).

As a caveat, however, it is important to recognize the limits of the comparisons between industries. Perhaps, the most overused and, my opinion, most tortured comparison is between the airline industry and the healthcare delivery system. Although health care is far from perfect, it strikes me as somehow hollow to compare the two with absolute fidelity. I wonder, for example, how many passengers would do well on a transcontinental flight if half boarded the flight with a preexisting medical condition, such as inpatients in a hospital. There are real and important nuances in the delivery of health care, in the training of the next generation of physicianscientists, and in performing the research that will inform the future treatment of patients. With such a caveat in mind, it is important to recognize that in an era of unprecedented fluidity, there may no longer be truly distinct disciplines.

\section{DECISION-MAKERS MUST POSSESS INTIMATE KNOWLEDGE OF THE PRODUCT}

In keeping with the overall notion that the knowledge of physicians have meaningful currency, providers must be invested with the power to meaningfully and durably inform the process. Certainly, the very people responsible for training the next generation of physicians must be invested with the power to shape the training experience. Similarly, crafting the next generation of physician-scientists, particularly those scientists with a focus on child health and disease, demands the input of the present generation of physician-scientists. We ought invite the best educational theorists, the most rigorous scientists, and the system engineers who can streamline our health care delivery models. However, even more certainly, we should not shrink away from our responsibility to inform the conversation, articulate the issues, and ensure that we, the academic physician-scientists with the most comprehensive, nuanced, and detailed understanding of the issues, are fully enfranchised in all aspects of the conversation. Our intimate knowledge of the desired product confers both power and substantial responsibility.

\section{CONCLUSION}

To fully realize our promise, academic pediatrics must continue to heed the lessons learned over the past 100 years, at the bedside of children, and as a field in evolution. It is my sincere hope that the present address provides five key points (Table 1) that warrant our collective attention as we seek our future ever while being ever mindful of our collective past.

First, clinical discoveries create important knowledge. The overall notion of moving from bedside to bench highlights the intrinsic value of clinical medicine wherein knowledge is created in a bidirectional manner from bed to cannon and bed to bench. Second, clinically driven knowledge discovery yields progress that is incremental. Although incremental, the process affords sustained and durable improvement. Third, narrative weds the past to the future and provides both context and a focus on humanity. The focus on humanity promotes a culture of deep, durable knowledge that places a high value on the primacy of individual relationships that ought inform all decisions that involve children and families. Fourth, it is

Table 1. Principles derived from the narrative of clinical discovery that might guide our path toward a stronger future for academic pediatrics

Discoveries create knowledge-bedside to bench

Values clinical medicine, bidirectional creation-bed to cannon, bed to bench

Clinically driven knowledge discovery yields incremental progress

Affords sustained and durable improvement

Narrative weds the past to the future, providing both context and focus on humanity

Promotes a culture of deep, durable knowledge, pediatrics-based decisions, and the primacy of individual relationships

Progress occurs at the margins of seemingly distinct disciplines

Physician-scientists, rigorous scientific training, medically conversant scientists are essential

Decision-makers must possess with intimate knowledge of the product Academic pediatricians must be invested in the decision-making process 
essential that we recognize that true progress occurs at the margins of seemingly distinct disciplines. To capitalize on the unprecedented rate of change and logarithmic increase in scientific knowledge and capabilities, we must continue to train and value physician-scientists with rigorous scientific training. Never before have medically conversant scientists been more critical to the long-term success of pediatrics. Finally, it is imperative that people with intimate knowledge of the product be invested with the power to meaningfully inform the decisions that will determine the training experiences, skill sets, and scientific capabilities of the next generation of physician scientists. There can be no substitute for academic pediatric representation at the decision-making table.

These sentiments are entirely consistent with the mission statement of The Society for Pediatric Research (SPR). The SPR exists to foster the research and career development of investigators engaged in creating new knowledge that advances the health and well being of children and youth. It has been my high privilege to serve as President of the SPR for the past year.

\section{REFERENCES}

1. Brandeis LD 1914 Business: a profession. In: Poole E (ed) Business: a profession Small, Maynard \& Co., Boston. pp 313-332

2. Haggerty RJ, Friedman SB 2003 History of developmental-behavioral pediatrics. J Dev Behav Pediatr 24:S1-S18

3. Wise PH 2009 The rebirth of pediatrics. Pediatrics 123:413-416

4. Guralnick S, Rushton J, Bale Jr JF, Norwood V, Trimm F, Schumacher D 2010 The response of the APPD, CoPS and AAP to the Institute of Medicine report on resident duty hours. Pediatrics 125:786-790

5. Landrigan CP, Fahrenkopf AM, Lewin D, Sharek PJ, Barger LK, Eisner M, Edwards S, Chiang VW, Wiedermann BL, Sectish TC 2008 Effects of the accreditation council for graduate medical education duty hour limits on sleep, work hours, and safety. Pediatrics 122:250-258

6. Ferkol T, Zeitlin P, Abman S, Blaisdell CJ, O'Brodovich H 2010 NHLBI training workshop report: the vanishing pediatric pulmonary investigator and recommendations for recovery. Pediatr Pulm 45:25-33

7. Grande JP 2009 Training of physicians for the twenty-first century: role of the basic sciences. Med Teach 31:802-806

8. Zambon M, Vincent JL 2008 Mortality rates for patients with acute lung injury/ ARDS have decreased over time. Chest 133:1120-1127

9. Moss RB 2009 Infection, inflammation, and the downward spiral of cystic fibrosis lung disease. J Pediatr 154:162-163

10. Ferkol T, Rosenfeld M, Milla CE 2006 Cystic fibrosis pulmonary exacerbations. J Pediatr 148:259-264

11. Laënnec RTH. 1821. A treatise on the diseases of the chest (English translation from the French). Preface and notes by Forbes J. T and G Underwood, London, pp 408

12. Stevens AA 1926 The Practice of Medicine. Saunders, Philadelphia

13. Brewer LA 3rd 1981 A historical account of the "wet lung of trauma" and the introduction of intermittent positive-pressure oxygen therapy in world war II. Ann Thorac Surg 31:386-393

14. Ashbaugh DG, Bigelow DB, Petty TL, Levine BE 1967 Acute respiratory distress in adults. Lancet 2:319-323

15. Josselson R, Lieblich A 1995 Interpreting experience: the narrative study of lives. Sage Publications, Inc., Thousand Oaks

16. Wilson ME, Megel ME, Enenbach L, Carlson KL 2010 The voices of children: stories about hospitalization. J Pediatr Health Care 24:95-102

17. Potts AL, Barr FE, Gregory DF, Wright L, Patel NR 2004 Computerized physician order entry and medication errors in a pediatric critical care unit. Pediatrics 113:59-63

18. Abowd GD, Anind KD, Brown PJ, Davies N, Smith M, Steggles P 1999 Towards a better understanding of context and context-awareness. In: Handheld and Ubiquitous Computing, Springer, pp 304-307

19. Alsos OA, Dabelow B, Faxvaag A 2011 Doctors' concerns of PDAs in the ward round situation. Lessons from a formative simulation study. Methods Inf Med 50:190-200

20. Fortuna RJ, Palfrey JS, Shelov SP, Samuels RC 2009 Paediatric experiences with work-hour limitations. Paediatric experiences with work-hour limitations. J Eval Clin Pract 15:116-120

21. Mihelcic JR, Crittenden JC, Small MJ, Shonnard DR, Hokanson DR, Zhang Q, Chen H, Sorby SA, James VU, Sutherland JW, Schnoor JL 2003 Sustainability science and engineering: the emergence of a new metadiscipline. Environ Sci Technol 37:53145324

22. Jiang N, Weinstein JA, Penland L, White RA, Fisher DS, Quake S 2011 Determinism and stochasticity during maturation of the zebrafish antibody repertoire. Proc Natl Acad Sci USA 108:5348-5353 